\title{
Plasma currents induced by resonant magnetic field perturbations in tokamaks
}

D. Reiser, and D. Chandra

Citation: Physics of Plasmas 16, 042317 (2009); doi: 10.1063/1.3126548

View online: https://doi.org/10.1063/1.3126548

View Table of Contents: http://aip.scitation.org/toc/php/16/4

Published by the American Institute of Physics

\section{Articles you may be interested in}

Non-linear magnetohydrodynamic modeling of plasma response to resonant magnetic perturbations Physics of Plasmas 20, 102510 (2013); 10.1063/1.4824820

The interaction of resonant magnetic perturbations with rotating plasmas

Physics of Fluids B: Plasma Physics 3, 644 (1991); 10.1063/1.859863

Bifurcated states of a rotating tokamak plasma in the presence of a static error-field

Physics of Plasmas 5, 3325 (1998); 10.1063/1.873000

Effects of resistivity on linear plasma responses to resonant magnetic perturbations in tokamak plasmas

Physics of Plasmas 23, 092502 (2016); 10.1063/1.4961919

Forced magnetic reconnection

The Physics of Fluids 28, 2412 (1985); 10.1063/1.865247

Edge localized modes and the pedestal: A model based on coupled peeling-ballooning modes

Physics of Plasmas 9, 2037 (2002); 10.1063/1.1449463

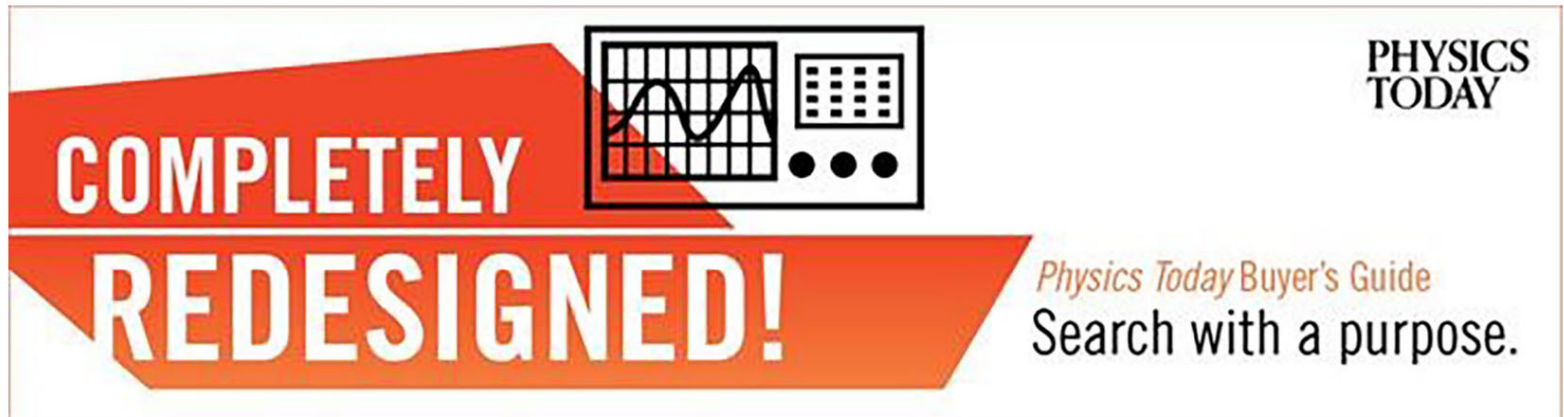




\title{
Plasma currents induced by resonant magnetic field perturbations in tokamaks
}

\author{
D. Reiser ${ }^{1}$ and D. Chandra ${ }^{2}$ \\ ${ }^{1}$ EURATOM Association, Institut für Energieforschung-Plasmaphysik, Forschungszentrum Jülich GmbH, \\ Trilateral Euregio Cluster, D-52425 Jülich, Germany \\ ${ }^{2}$ Institute for Plasma Research, Bhat, Gandhinagar 382428, India
}

(Received 10 February 2009; accepted 31 March 2009; published online 30 April 2009)

\begin{abstract}
The plasma response on externally applied resonant magnetic field perturbations is studied by means of numerical simulations. It is shown that dependent on collisionality and perturbation strength, plasma currents build up which can compensate the external field. These plasma currents are accompanied by out-of-phase currents and poloidal flows at the resonant surfaces. With an increasing perturbation field the screening of the externally applied field decreases and at a certain level, the vacuum field approximation holds for the total magnetic field. () 2009 American Institute of Physics. [DOI: 10.1063/1.3126548]
\end{abstract}

\section{INTRODUCTION}

Resonant magnetic field perturbations (RMPs) are known to significantly change the magnetic field topology and therefore the plasma dynamics in a tokamak device. ${ }^{1-7}$ Such resonant perturbations are, in general, characterized by a magnetic field component perpendicular to the flux surfaces and a zero derivative along the unperturbed magnetic field lines at particular (resonant) flux surfaces. They can be caused by imperfections in the coil arrangements or by means of additional RMP coils to introduce deliberately large deviations from the usual axisymmetric tokamak confinement field. Ever since it is known that RMPs can support an efficient control of edge localized modes for certain operational windows, in particular, the use of RMP coil arrangements has attracted increasing interest. ${ }^{8-10}$

Effects due to the presence of RMPs have been studied theoretically and numerically on the basis of kinetic and fluid models on different levels of complexity, one important aspect of it being the structure of self-consistent currents induced in the plasma which might cause significant changes in the magnetic field topology. A widely used assumption in theoretical and numerical studies is the vacuum approximation, ${ }^{11-15}$ which considers the total magnetic field in the plasma region as a sum of the axisymmetric confinement field and the vacuum field induced by the external coils. This vacuum approximation neglects a possible screening of the RMP field and the related change in the topology. More sophisticated approaches including plasma currents often employ the so-called magnetohydrodynamics (MHD) approximation which assumes particularly that pressure forces on the electrons cannot compete with the electric field. ${ }^{16,17}$ Other approaches consider cylindrical geometry and the plasma response of basic resonant modes only. ${ }^{18,19}$ More complete models have been analyzed in Refs. 20-24 but are also restricted to slab or cylinder geometry. In this paper the occurrence of self-consistent screening currents in the framework of a four-field drift-fluid model ${ }^{25-30}$ is studied. This nonlinear four-field version of the dissipative drift-Alfvén model includes electromagnetic plasma dynamics, avoids the
MHD approximation and takes into account curvature effects due the toroidicity of the equilibrium magnetic field. The numerical simulations are performed with the ATTEMPT $\operatorname{code}^{30}$ for a variety of plasma scenarios quite close to TEXTOR-like parameters. ${ }^{2}$ Special attention will be paid to the collisionality dependence and the occurrence of a drop in the screening efficiency with increasing RMP field. It is shown that shielding currents are induced in the presence of RMPs which can be strong enough to screen the RMP field completely. The screening is increased if $\hat{\beta}$, the scaled plasma beta, is increased and decreased if $\hat{C}$, the scaled collisionality, is increased. With increasing RMP field strength a nonlinear effect leads to a drop of screening efficiency. This nonlinear effect is manifest in a build-up of a static and axisymmetric radial electric field until it compensates the force due to the radial pressure gradient. Thus, the screening currents are accompanied by zonal flows at the resonant surfaces. In addition, an out-of-phase current generally occurs which is coupled to geodesic acoustic mode (GAM) dynamics. These numerical results are in qualitative agreement with experimental findings on poloidal rotation and GAM dynamics. ${ }^{31-34}$ The paper is structured in the following order: In Sec. II basic features of the drift-fluid model used for the numerical investigations and its extension for RMP studies are recapitulated; in Sec. III the numerical experiment is specified and in Sec. IV simulation results are presented for a variety of computations; in Sec. V the numerical results are discussed and supplemented by means of simplified balance equations to describe the new static plasma state in the presence of the RMPs; finally Sec. VI summarizes the results with special emphasis on the relevance of the screening effects for standard tokamak operational windows.

\section{DRIFT-FLUID MODEL WITH RMP FIELDS}

In this section the basic features of the drift-fluid model used in the numerical investigations are outlined. The model describes the deviations of particle density $n$, electric potential $\phi$, magnetic potential $A$, and parallel ion flow velocity $u_{\|}$ 
from an equilibrium state. This equilibrium state is prescribed by a constant background density gradient $\nabla n_{0}$, but equilibrium flows and equilibrium electric fields are not prescribed. The electric fields evolve self-consistently in the model, and the resulting statistically stationary and axisymmetric piece is considered as the equilibrium profile. Electron temperature dynamics is neglected $\left(T_{e}=\right.$ const $)$ and cold ions $\left(T_{i}=0\right)$ are assumed. The equations for particle balance, the quasineutrality condition $(\boldsymbol{\nabla} \cdot \mathbf{J}=0)$, Ohm's law, and the parallel ion momentum balance read

$$
\begin{aligned}
\frac{\partial n}{\partial t}= & -\mathbf{v}_{E} \cdot \nabla\left(n_{0}+n\right)+\frac{1}{e} \nabla_{\|} J_{\|}-n_{0} \nabla_{\|} u_{\|}-n_{0} \mathcal{K}(\phi) \\
& +\frac{T_{e}}{e} \mathcal{K}(n),
\end{aligned}
$$

$$
\frac{n_{0} m_{i}}{B_{0}^{2}} \frac{\partial w}{\partial t}=-\frac{n_{0} m_{i}}{B_{0}^{2}} \mathbf{v}_{E} \cdot \nabla w+\nabla_{\|} J_{\|}+T_{e} \mathcal{K}(n),
$$

$$
\frac{\partial A}{\partial t}+\frac{m_{e}}{e^{2} n_{0}} \frac{\partial J_{\|}}{\partial t}=-\frac{m_{e}}{e^{2} n_{0}} \mathbf{v}_{E} \cdot \nabla J_{\|}+\frac{T_{e}}{e n_{0}} \nabla_{\|} n-\nabla_{\|} \phi-\eta_{\|} J_{\|},
$$

$$
n_{0} m_{i} \frac{\partial u_{\|}}{\partial t}=-n_{0} m_{i} \mathbf{v}_{E} \cdot \nabla u_{\|}-T_{e} \nabla_{\|} n,
$$

with auxiliary relations for the vorticity $w$ and the parallel current density $J_{\|}$,

$$
w=\nabla_{\perp}^{2} \phi, \quad \mu_{0} J_{\|}=-\nabla_{\perp}^{2} A .
$$

The operators are defined by

$$
\begin{aligned}
& \frac{d}{d t}=\frac{\partial}{\partial t}+\mathbf{v}_{E} \cdot \nabla, \\
& \mathbf{v}_{E} \cdot \nabla f=\frac{\mathbf{B}}{B^{2}} \cdot(\nabla \phi \times \nabla f), \\
& \mathcal{K}(f)=\nabla \cdot\left(\frac{\mathbf{B} \times \boldsymbol{\nabla} f}{B^{2}}\right), \\
& \nabla_{\|} f=\frac{\mathbf{B}}{B} \cdot \nabla f-\frac{\mathbf{B}}{B^{2}} \cdot(\nabla A \times \nabla f), \\
& \nabla_{\perp}^{2}=\nabla^{2} f-\nabla \cdot \frac{\mathbf{B}}{B} \frac{\mathbf{B}}{B} \cdot \nabla f .
\end{aligned}
$$

Here $\mathbf{B}$ denotes the equilibrium magnetic field, $e$ is the elementary charge, $\eta_{\|}$is the classical parallel resistivity, ${ }^{35}$ and $m_{e}$ and $m_{i}$ are the electron and ion mass, respectively. The model is local, i.e., the background density $n_{0}$, the reference magnetic field $B_{0}$, and the electron temperature $T_{e}$ are treated as constants, except in the background density gradient $\nabla n_{0}$ which is also considered as constant. A detailed discussion of the basic physics described by the model can be found in Ref. 25 and references therein. The magnetic equilibrium field is assumed to consist of nested circular flux surfaces representing the plasma region just inside the last closed flux surface in a toroidal geometry with large aspect ratio. Therefore

$$
\mathbf{B}=\frac{B_{0}}{q R} \mathbf{e}_{\theta}+\frac{B_{0} R_{0}}{R^{2}} \mathbf{e}_{\varphi},
$$

where $R_{0}$ is the tokamak major radius and $R=R_{0}+r \cos \theta$ is the distance from the torus axis. The $q$-profile is assumed to be linear $q=q_{0}+q_{0} \hat{s}\left(r-r_{0}\right) / r_{0}$, with magnetic shear $\hat{s}$ and reference minor radius $r_{0}$. The transformation between the Cartesian coordinates $\left(x_{1}, x_{2}, x_{3}\right)$ and the toroidal coordinates $(r, \theta, \varphi)$ is given by

$$
x_{1}=R \cos \varphi, \quad x_{2}=R \sin \varphi, \quad x_{3}=-r \sin \theta .
$$

The additional transformations

$$
s=\theta, \quad x=\frac{r-r_{0}}{\rho_{s}}, \quad y=\frac{r_{0}}{q_{0} \rho_{s}} \quad(q \theta-\varphi),
$$

and

$$
\sigma=\theta, \quad \chi=\frac{r-r_{0}}{\rho_{s}}, \quad \eta=\frac{r_{0}}{q_{0} \rho_{s}} \quad\left(q_{0} \theta-\varphi\right)
$$

introduce field aligned coordinates to simplify the analysis for flute approximation $\left(k_{\|}<k_{\perp}\right)$ and the numerical implementation. Here $s$ is a parallel coordinate, field aligned in the entire domain, and $\sigma$ is field aligned at the reference surface at $r=r_{0}$ only. These definitions allow to rewrite the (conveniently scaled) operators in flute approximation as follows:

$$
\begin{aligned}
& \mathbf{v}_{E} \cdot \nabla f=\frac{\partial \phi}{\partial \chi} \frac{\partial f}{\partial \eta}-\frac{\partial \phi}{\partial \eta} \frac{\partial f}{\partial \chi}, \\
& \mathcal{K}(f)=-\omega_{B}\left(\cos s \frac{\partial f}{\partial \eta}+\sin s \frac{\partial f}{\partial \chi}\right), \\
& \nabla_{\|} f=\frac{\partial f}{\partial s}-\hat{\beta}\left(\frac{\partial A}{\partial \chi} \frac{\partial f}{\partial \eta}-\frac{\partial A}{\partial \eta} \frac{\partial f}{\partial \chi}\right), \\
& \nabla_{\perp}^{2} f=\frac{\partial^{2} f}{\partial \chi^{2}}+\frac{\partial^{2} f}{\partial \eta^{2}} .
\end{aligned}
$$

Thus, one obtains the following normalized version of Eqs. (1)-(5):

$$
\begin{aligned}
& \frac{\partial n}{\partial t}=-\{\phi, n\}-\mathcal{K}(\phi-n)+\nabla_{\|}\left(J_{\|}-u_{\|}\right), \\
& \frac{\partial w}{\partial t}=-\{\phi, w\}+\mathcal{K}(n)+\nabla_{\|} J_{\|}, \\
& \hat{\beta} \frac{\partial A}{\partial t}+\hat{\mu} \frac{\partial J_{\|}}{\partial t}=-\hat{\mu}\left\{\phi, J_{\|}\right\}+\nabla_{\|}(n-\phi)-\hat{C} J_{\|}, \\
& \hat{\epsilon} \frac{\partial u_{\|}}{\partial t}=-\hat{\epsilon}\left\{\phi, u_{\|}\right\}-\nabla_{\|} n,
\end{aligned}
$$

where 


$$
w=\nabla_{\perp}^{2} \phi, \quad J_{\|}=-\nabla_{\perp}^{2} A .
$$

The dimensionless form of the local model equations (19)-(23) forms the basis for the numerical investigations. The transformation to the coordinates used here is explained in detail in Ref. 30. The remaining set of dimensionless model parameters are considered as constants in the local model,

$$
\begin{aligned}
& \hat{\epsilon}=\left(\frac{q_{0} R_{0}}{L_{\perp}}\right)^{2}, \quad \hat{\beta}=\hat{\epsilon} \beta, \quad \hat{\mu}=\hat{\epsilon} \frac{m_{e}}{m_{i}}, \\
& \hat{C}=\hat{\mu} \frac{L_{\perp}}{c_{s}} \frac{\nu_{e}}{1.96}, \quad \omega_{B}=2 \frac{L_{\perp}}{R_{0}},
\end{aligned}
$$

where

$$
\begin{aligned}
& \beta=\frac{\mu_{0} n_{0} T_{e}}{B_{0}^{2}}, \quad \rho_{s}=\frac{c_{s} m_{i}}{e B_{0}}, \\
& c_{s}=\sqrt{\frac{T_{e}}{m_{i}}}, \quad \nu_{e}=\frac{e^{4} n_{0} \ln \Lambda}{3(2 \pi)^{3 / 2} \epsilon_{0}^{2} m_{e}^{1 / 2} T_{e}^{3 / 2}} .
\end{aligned}
$$

In detail these are the ballooning parameter $\omega_{B}$, representing the curvature effect, due to toroidicity, i.e., the $1 / R$-dependence of the equilibrium toroidal magnetic field. Furthermore, the ratio $\hat{\epsilon}$ of parallel scale length $q_{0} R_{0}$ and perpendicular background density scale length $L_{\perp}$, the scaled plasma beta $\hat{\beta}$, the scaled mass ratio $\hat{\mu}$ of electron and ion mass and the scaled electron collision frequency $\hat{C}$. The curvature effect due to toroidicity provides a coupling of poloidal modes. It is to be noted that a mixed representation is used in the formulation of Eqs. (19)-(23), i.e., $s, \chi$, and $\eta$ do not constitute a single coordinate system. The constant background density gradient is $\partial n_{0} / \partial \chi=-1$ in scaled units, which defines the scale length $L_{\perp}$. Finally it is to be noted that Eqs. (19)-(23) are completed by dissipative processes via the extension

$$
\frac{\partial f}{\partial t}=S+\mu_{\perp} \nabla_{\perp}^{2} f+\mu_{\|} \nabla_{\|}^{2} f,
$$

where $f=n, w, u$, and $S$ are the respective right hand side of Eqs. (19)-(22). The dissipation coefficients $\mu_{\perp}$ and $\mu_{\|}$are prescribed in an appropriate range to provide sufficient dissipation for obtaining a saturated plasma state, but without disturbing the basic physics to be studied. As in previous studies the perturbation field is included by the replacement $A \rightarrow A+A^{*}$ in the model equations, ${ }^{29}$ where

$$
\begin{aligned}
A^{*} & =\sum_{m_{*}=-1}^{1} A_{0} e^{n_{*} k_{\eta} \chi} \cos \left(m_{*} \sigma+n_{*} k_{\eta} \eta\right) \\
& =\sum_{m_{*}=-1}^{1} A_{0} e^{\left[q_{0}\left(r-r_{0}\right) / L_{\perp}\right]} \cos \left[\left(m_{*}+n_{*} q_{0}\right) \theta-n_{*} \varphi\right],
\end{aligned}
$$

representing a current free RMP field, i.e., $\nabla_{\perp}^{2} A^{*}=0$, with resonances at $q=\left(m_{*}+n_{*} q_{0}\right) / n_{*}$. In this work RMPs with toroidal mode number $n_{*}=2$ are considered, representing a static externally induced perturbation field consisting of the three modes with poloidal/toroidal mode numbers 5/2, $6 / 2$, and $7 / 2$, representative for TEXTOR-DED operation.

\section{NUMERICAL EXPERIMENT}

For the numerical simulation the computational grid is defined in the $(\sigma, \chi, \eta)$-coordinates with grid dimensions in scaled units as

$$
L_{\sigma}=2 \pi, \quad L_{\chi}=\frac{N_{\chi}}{N_{\eta}} \frac{L_{\eta}}{\pi \hat{s}}, \quad L_{\eta}=\frac{2 \pi r_{0}}{q_{0} \rho_{s}} .
$$

The number of grid points is $N_{\sigma} \times N_{\chi} \times N_{\eta}=16 \times 128 \times 128$. The following set of parameters has been chosen to get close to TEXTOR-like plasma conditions. The entire flux surfaces in the range $2 \leq q \leq 4$ around the reference radius $r_{0}$ $=0.5 \mathrm{~m}$ are considered, with $q_{0}=q\left(r_{0}\right)=3$ and magnetic shear $\hat{s}=1$. This corresponds to a radial extent of $0.34 \mathrm{~m}$ $\leq r \leq 0.66 \mathrm{~m}$. The dimensionless model parameters are chosen as $\omega_{B}=0.046, \hat{\mu}=4.7$, and $\hat{\epsilon}=17227$. The parameters $\hat{C}$ and $\hat{\beta}$ are varied in the range $0.5 \leq \hat{\beta}, \hat{C} \leq 5$.0. This represents roughly a plasma with physical parameters $R_{0}$ $=1.75 \mathrm{~m}, m_{i}=2 m_{p}, B_{0}=1 \mathrm{~T}, L_{\perp}=0.04 \mathrm{~m}$, and the following combination of densities and temperatures:

\begin{tabular}{cccr}
\hline$\hat{C}$ & $\hat{\beta}$ & $\begin{array}{c}n_{0} \\
\left(\mathrm{~m}^{-3} \times 10^{19}\right)\end{array}$ & $\begin{array}{c}T_{e} \\
(\mathrm{eV})\end{array}$ \\
\hline 0.5 & 0.5 & 0.3 & 52 \\
5.0 & 0.5 & 0.6 & 24 \\
5.0 & 5.0 & 2.8 & 52 \\
0.5 & 5.0 & 1.3 & 112 \\
\hline
\end{tabular}

The scans over RMP field strength are done in the range $1 \times 10^{-7} \mathrm{Tm} \leq A_{0} \leq 3 \times 10^{-5} \mathrm{Tm}$. The dissipation parameters are prescribed as $\mu_{\perp}=\mu_{\|}=0.01$ and the time step is $\Delta t=0.02 L_{\perp} / c_{s}$. It is to be noted that the corresponding unscaled dissipation coefficients $D_{\perp}$ and $D_{\|}$and the classical dissipation coefficients ${ }^{23}$

$$
D_{\perp}^{c}=4.7 \frac{T_{e} m_{e} \nu_{e}}{e^{2} B_{0}^{2}}, \quad D_{\|}^{c}=3.2 \sqrt{\frac{m_{e}}{m_{i}}} \frac{T_{e}}{m_{e} \nu_{e}}
$$

are related by

$$
\frac{D_{\perp}}{D_{\perp}^{c}} \approx 200 \frac{\hat{\mu}}{\hat{C}} \mu_{\perp}, \quad \frac{D_{\|}}{D_{\|}^{c}} \approx 40 \hat{C} \mu_{\|}
$$

for $m_{i}=2 m_{p}$. Thus, the perpendicular dissipation used is about a factor of 10 higher than the classical diffusion, and the parallel dissipation coefficient in the simulations is of the same order as the classical one. The radial boundary conditions used in the computations are Dirichlet zero for all dependent scalar fields $n, \phi, w, A, J_{\|}$, and $u_{\|}$. The simulation starts with a small random perturbation in the density $n$. Linear instabilities drive the evolution of a dynamical plasma state and the computation is continued until a nonlinearly saturated, i.e., statistically stationary, state is established. This final plasma state is analyzed by means of time averag- 

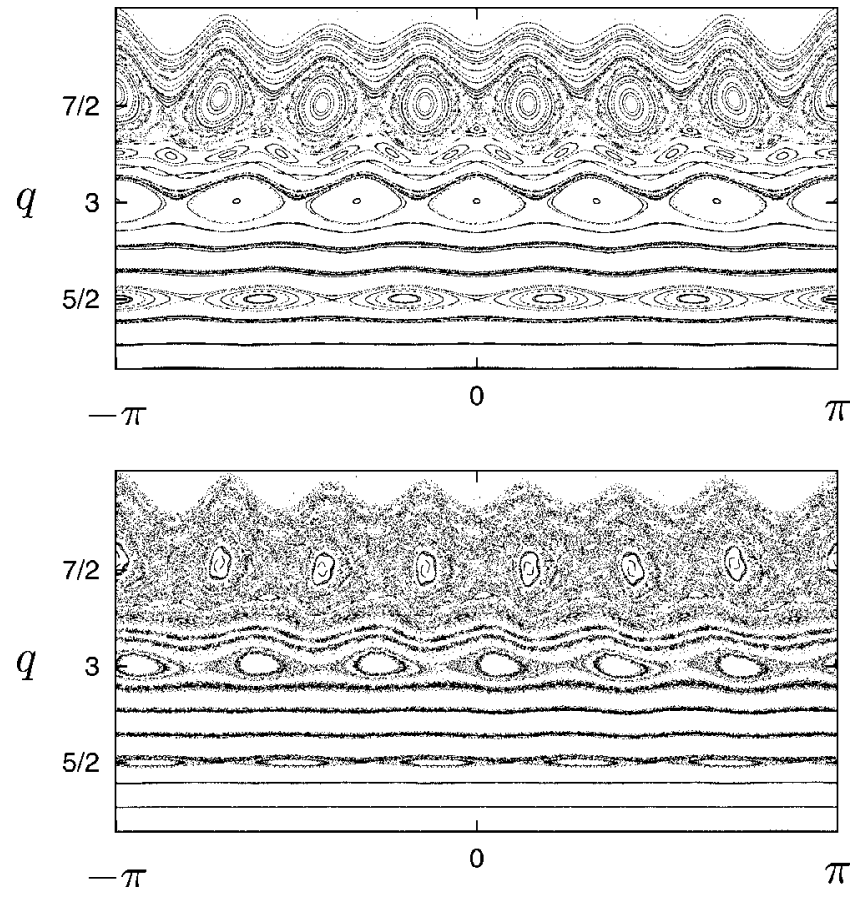

FIG. 1. Illustration of the screening effect for the $6 / 2$-configuration of a TEXTOR-DED-like geometry for the parameters $\hat{\beta}=5.0$ and $\hat{C}=2.5$. The $5 / 2$ - and 6/2-island chains of the vacuum field disappeared completely and have been replaced by islands shifted by $\pi / 2$ in toroidal direction due to out-of-phase currents.

ing over an appropriate interval to obtain the information about the new equilibrium.

\section{COMPUTATIONAL RESULTS}

In this section, results of numerical simulations performed in the parameter range sketched in Sec. III are presented. In order to solve the model equations (19)-(23) the time-dependent three-dimensional (3D) ATTEMPT $\operatorname{code}^{30}$ has been employed. Basic features of the screening effect in the presence of RMP fields are elucidated and analyzed by means of parameters scans varying the collisionalitity $\hat{C}$, the scaled beta $\hat{\beta}$, and the RMP field strength. In addition, certain simulation results are compared to results of reduced models in order to clarify the importance of particular physical mechanisms. Basis quantities of interest are the in-phase and out-of-phase currents, the static zonal flows and their relation to the screening efficiency.

It is shown that (1) plasma response currents can build up strong enough to screen RMP fields completely. (2) The screening is increased if $\hat{\beta}$ is increased and decreased if $\hat{C}$ is increased. (3) A nonlinear effect leads to a breakdown of screening efficiency with increasing RMP field strength. The nonlinear effect is manifested also in a static radial electric field rising until it compensates the radial pressure gradient. (4) The screening effect is predominantly caused by pressure effects, i.e., a competition of pressure forces and electric fields. However also curvature effects show a significant contribution by increasing the shielding of the penetrating RMP field.

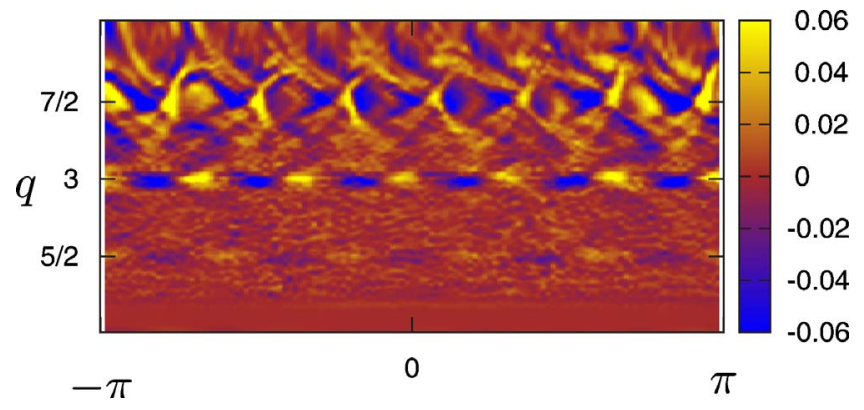

FIG. 2. (Color online) Pattern of the time averaged current density corresponding to the results of Fig. 1.

First an example of the RMP screening effect is presented, demonstrating the occurrence of significant plasma currents and their implications for the magnetic field topology. Then, in a second step, results from parameter scans illustrate the dependence of the screening efficiency on plasma parameters and RMP field strength. The section is completed with results obtained by the use of reduced models, where in particular the cylinder approximation and the neglect of pressure forces are considered.

\section{A. In-phase and out-of-phase currents}

An example for the induction of plasma countercurrents is given in Figs. 1 and 2. These results are obtained by simulations for $\hat{\beta}=5.0, \hat{C}=2.5$, and $A_{0}=2 \times 10^{-5} \mathrm{Tm}$. The top figure of Fig. 1 shows a Poincaré plot of the vacuum field according to Eq. (29). The bottom figure illustrates the structure of the total magnetic field, in which the plasma response, i.e., the self-consistent magnetic potential $A$ is also taken into account. The two-dimensional pattern of Fig. 2 shows the corresponding profile of the current density $J_{\|}$as a function of the poloidal angle $\theta$ and $q$ in the same poloidal plane as considered in Fig. 1. It can be seen that in the total magnetic field, the RMP vacuum field contribution is completely screened at the 5/2- and 6/2-surfaces, i.e., the island structures opened by the RMP field disappeared due to the build-up of a current density of symmetry $\cos (5 \theta-2 \varphi)$ and $\cos (6 \theta-2 \varphi)$, respectively. The 5/2- and 6/2-islands which are visible in the Poincaré plot are shifted by $\pi / 2$ and result from a current density of symmetry $\sin (5 \theta-2 \varphi)$ and $\sin (6 \theta$ $-2 \varphi$ ), respectively. In all simulations for this study such "out-of-phase currents" accompany the "in-phase" currents, being responsible for the screening of the RMP field. Both currents are well localized at the respective resonant surfaces, but spread out for increasing RMP field strength. Thus, the RMP field structure is partly diminished and replaced by a shifted structure of similar symmetry. An additional important consequence of these plasma currents is that the out-ofphase component couple to GAMs via Maxwell stress and therefore are able to significantly change their frequency and amplitude. $^{34}$ 

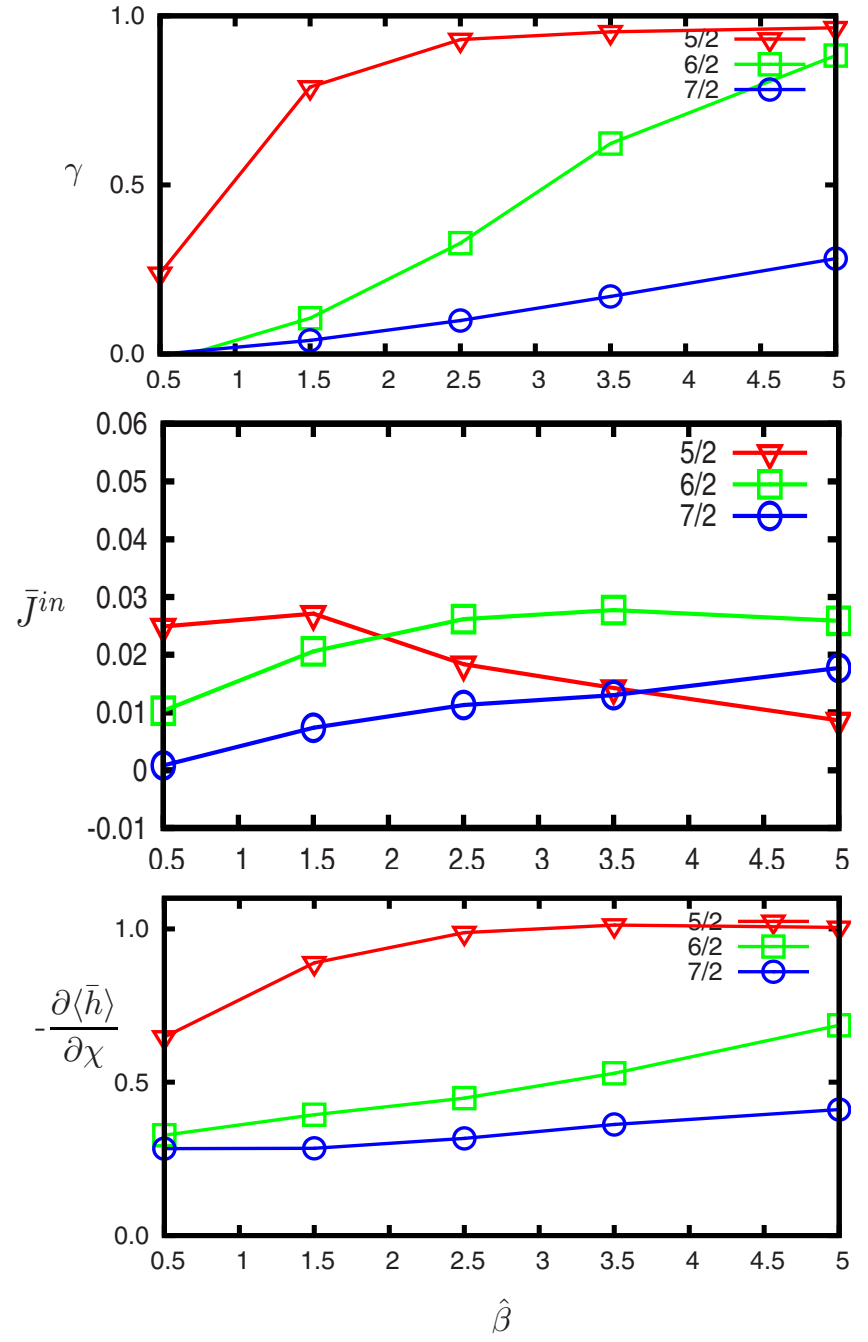

FIG. 3. (Color online) Screening efficiency at the respective resonant surfaces of the 5/2-, 6/2-, and 7/2-modes vs $\hat{\beta}$ for $\hat{C}=2.5$ and $A_{0}^{*}=2$ $\times 10^{-7} \mathrm{Tm}$

\section{B. Screening efficiency versus $\hat{\boldsymbol{\beta}}$ and $\hat{\boldsymbol{C}}$}

For a fixed RMP field strength of $A_{0}=2 \times 10^{-5} \mathrm{Tm}$, the values of $\hat{\beta}$ and $\hat{C}$ have been varied. In Figs. 3 and 4 the screening efficiencies $\gamma_{m, n}=-\operatorname{Re}\left(\bar{A}_{m, n}\right) / \operatorname{Re}\left(\bar{A}_{m, n}^{*}\right)$, the time averaged countercurrent density $\bar{J}_{m, n}^{\mathrm{n}}=\left\langle\bar{J}_{\|} \cos (m \theta-n \varphi)\right\rangle$, and the time averaged radial derivative of the axisymmetric component of the force $\partial\langle\bar{h}\rangle / \partial \chi=\partial\left\langle n_{0}+\bar{n}-\bar{\phi}\right\rangle / \partial \chi$ are shown. Here the brackets $\langle\ldots\rangle$ denote a flux surface average and the bar denotes a time average, taken over the diagnosis computational time interval. The Fourier components $\bar{A}_{m, n}, \bar{A}_{m, n}^{*}$, and $\bar{J}_{m, n}^{\text {in }}$ are taken at the respective resonant flux surfaces $q$ $=5 / 2,6 / 2$, and $7 / 2$ and are compared to the corresponding values of $\partial\langle\bar{h}\rangle / \partial \chi$. The screening efficiency $\gamma_{m, n}$ is a measure for the shielding of the radial component of the RMP field, responsible for island formation. Thus, a value of $\gamma_{m, n}=1$ means that the original $m / n$-island chain (RMP vacuum field) is completely suppressed at the resonant surface. A value $\gamma_{m, n}=0$ describes the case where the magnetic field is determined just by the vacuum structure of the RMP field,
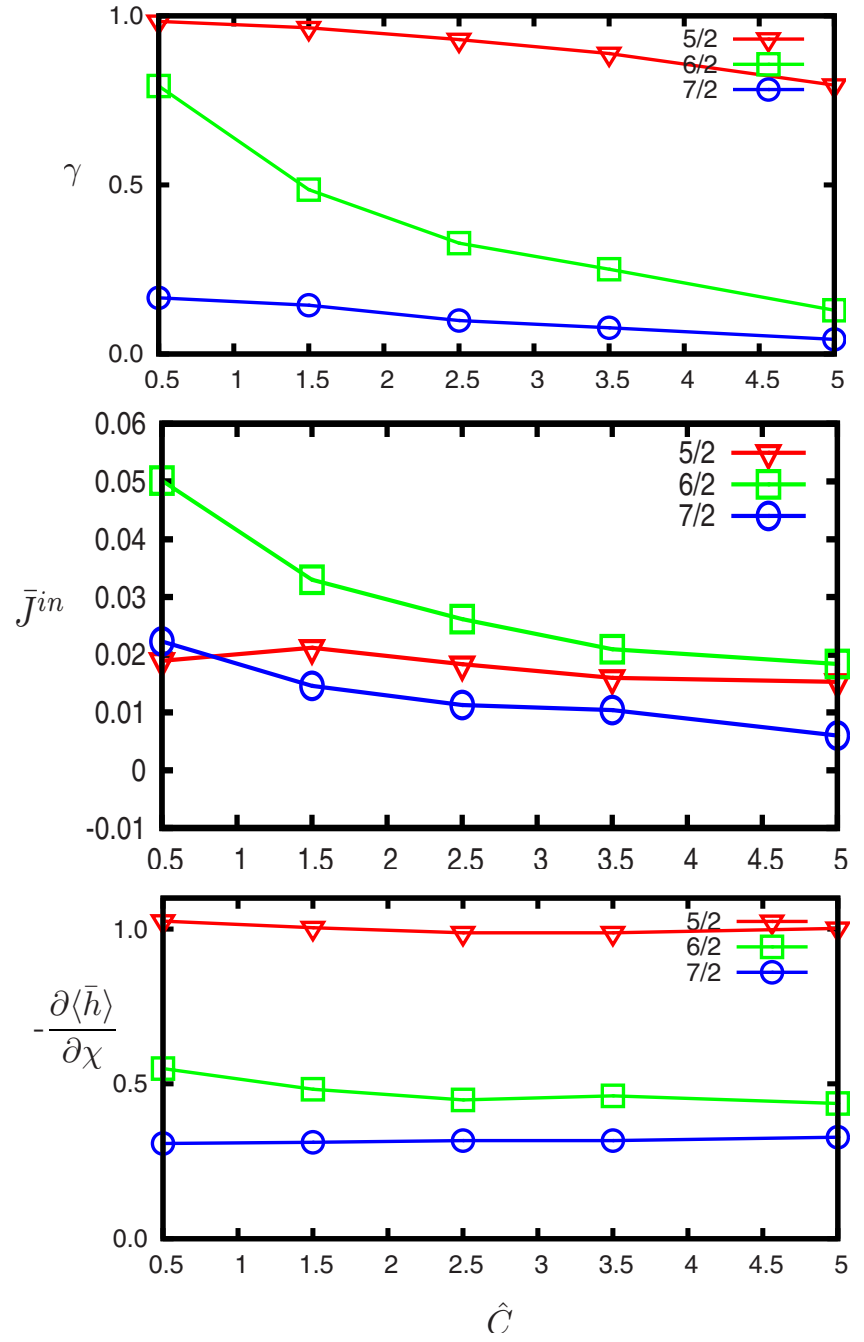

FIG. 4. (Color online) Screening efficiency at the respective resonant surfaces of the 5/2-, 6/2-, and 7/2-modes vs $\hat{C}$ for $\hat{\beta}=2.5$ and $A_{0}^{*}=2$ $\times 10^{-7} \mathrm{Tm}$.

i.e., complete penetration. In Fig. 3, top, the screening efficiency as a function of $\hat{\beta}$ shows a clear increase with increasing $\hat{\beta}$. In particular the innermost resonance at $q=5 / 2$ goes quickly into complete screening. The corresponding current densities, shown in the midfigure, reflect this trend-at least for the mode numbers $6 / 2$ and $7 / 2$. However, a much more obvious correlation with the screening efficiency is found for the force balance $\partial\langle\bar{h}\rangle / \partial \chi$. Significant variations of these quantities can be observed also in Fig. 4, but there the screening is reduced for increasing $\hat{C}$. Again the resonant currents for $m / n=6 / 2$ and $7 / 2$ follow the trends of $\gamma_{6,2}$ and $\gamma_{7,2}$ but $\partial\langle\bar{h}\rangle / \partial \chi$ shows an even more pronounced correlation.

\section{Screening efficiency versus RMP field strength}

In Fig. 5 results are shown for $\hat{\beta}=5.0$ and $\hat{C}=5.0$ and parameter $A_{0}$ varied in the range $1 \times 10^{-7} \mathrm{Tm} \leq A_{0} \leq 3$ $\times 10^{-5} \mathrm{Tm}$. The screening efficiency displays an obvious trend to be decreased by increasing RMP field strength. In particular, the screening of the 6/2- and 7/2-modes exhibits a 

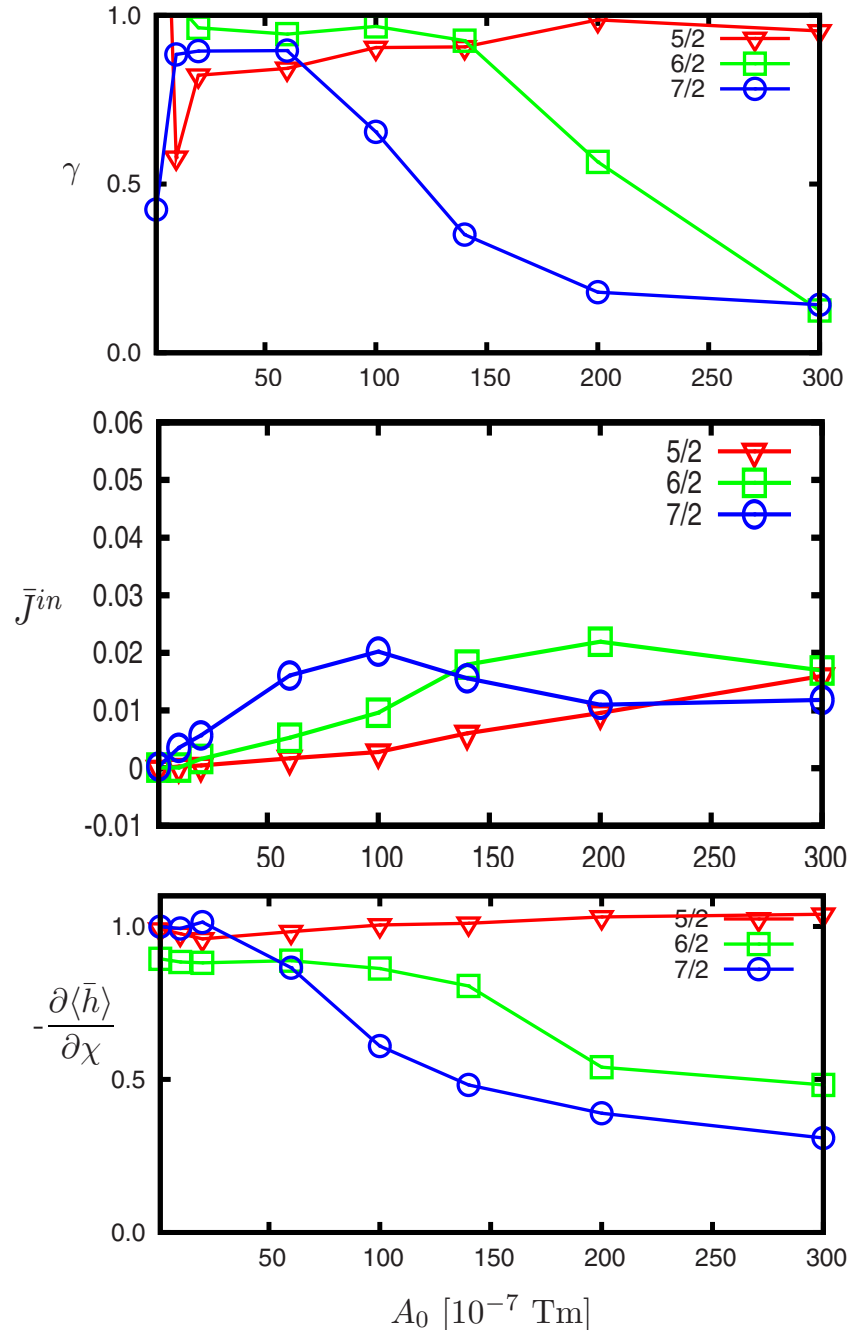

FIG. 5. (Color online) Screening efficiency at the respective resonant surfaces of the 5/2-, 6/2-, and 7/2-modes vs RMP field strength for $\hat{\beta}=5.0$ and $\hat{C}=5.0$.

quite abrupt drop in a certain range of $A_{0}$. The corresponding countercurrents are first increased with increasing $A_{0}$ but are reduced again for higher RMP fields. As in the scans over $\hat{\beta}$ and $\hat{C}$ the correlation between $\gamma_{m, n}$ and $\partial\langle\bar{h}\rangle / \partial \chi$ is striking. It has to be noted that even though the background density gradient $\partial\left\langle n_{0}\right\rangle / \partial \chi=-1$ is prescribed, a certain density profile flattening occurs in the computation. However the strongest contribution to the variation of $\partial\langle\bar{h}\rangle / \partial \chi$ stems from the changes in the radial electric field. Consequently a zonal flow is generated by the RMP up to a level where the electric field compensates the pressure gradient of the background plasma. The reduced screening is manifest in the force compensation $\partial\langle\bar{h}\rangle / \partial \chi \rightarrow 0$. The delay in the shielding of the different components of the model RMP can be explained by the fact that the perturbation field is exponentially decaying. Thus, the 7/2-surface is located at higher RMP level, i.e., the transition effect first appears for the corresponding resonant 7/2-mode in the $A_{0}$-scan. An additional plot of the different currents versus the local RMP field strength are shown in

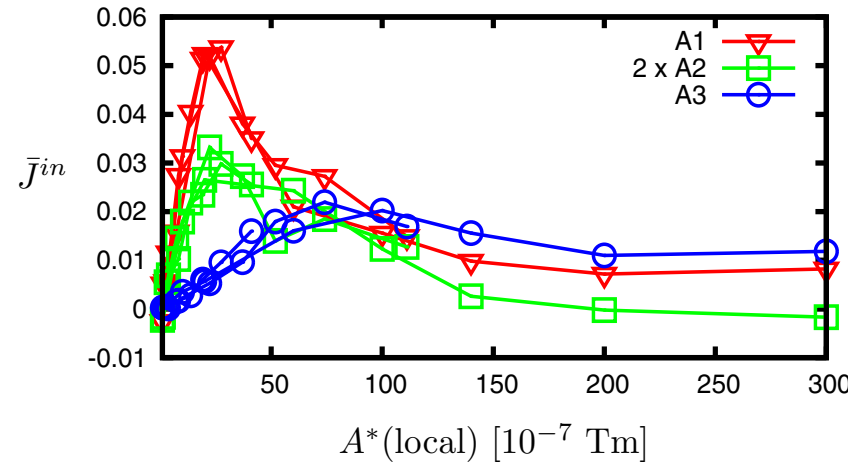

FIG. 6. (Color online) In-phase currents at the resonant surfaces vs the local RMP field amplitude from simulation (top) and obtained by half-analytical estimate equation (33) (bottom) for $\hat{\beta}=0.5, \hat{C}=0.5$ (case A1), $\hat{\beta}=0.5, \hat{C}$ $=5.0($ case A2) and $\hat{\beta}=5.0, \hat{C}=5.0($ case A $)$.

Fig. 6 and this proves that the plasma response is guided just by the local magnetic field and that the different resonant modes follow a very similar trend.

\section{Impact of island overlap}

As mentioned in the previous section, the delay in the plasma response at the different resonant surfaces with increasing RMP level is caused mainly by the exponential radial decay of the RMP field. In order to illustrate this observation, Fig. 6 shows the superposition of the in-phase currents at $q=5 / 2,6 / 2$, and $7 / 2$ as a function of the local effective RMP field amplitude at the respective surface. Three cases are shown, representing simulations with $\hat{\beta}$ $=0.5, \hat{C}=0.5$ (case A1), $\hat{\beta}=0.5, \hat{C}=5.0$ (case A2), and $\hat{\beta}$ $=5.0, \hat{C}=5.0$ (case A3). It can be seen that the plasma response currents behave very similar at the different resonant surfaces. Thus, the overlap of the RMP island chains (measured often by the Chirikov parameter) is obviously not of importance for the particular screening effect considered here.

\section{E. Comparison with reduced models}

In order to clarify which effects might be dominant or negligible in the build-up of the shielding currents a number of simulations have been repeated with particular physics switched off. Three variations have been considered: (i) the parallel ion flow has been neglected, i.e., $u_{\|}=0$, (ii) the curvature effect has been switched off, i.e., the geometry is reduced to a cylinder by $\mathcal{K}(n)=\mathcal{K}(\phi)=0$, and (iii) the so-called MHD approximation has been used, i.e., the terms $\nabla_{\|} J_{\|}$in the density balance and $\nabla_{\|} n$ in Ohm's law have been set to zero. In Fig. 7, top, the results for case (i) are shown and no significant difference can be found compared to the full model simulations in Fig. 5. Case (ii), midfigure of Fig. 7, on the other hand, gives a reduced screening efficiency, where the drop in the screening appears for much lower RMP field amplitudes than observed in the previous results. The conclusion to be drawn is that curvature effects additionally enhance the screening effect. However, the most striking difference comes with the neglect of pressure effects, illustrated 

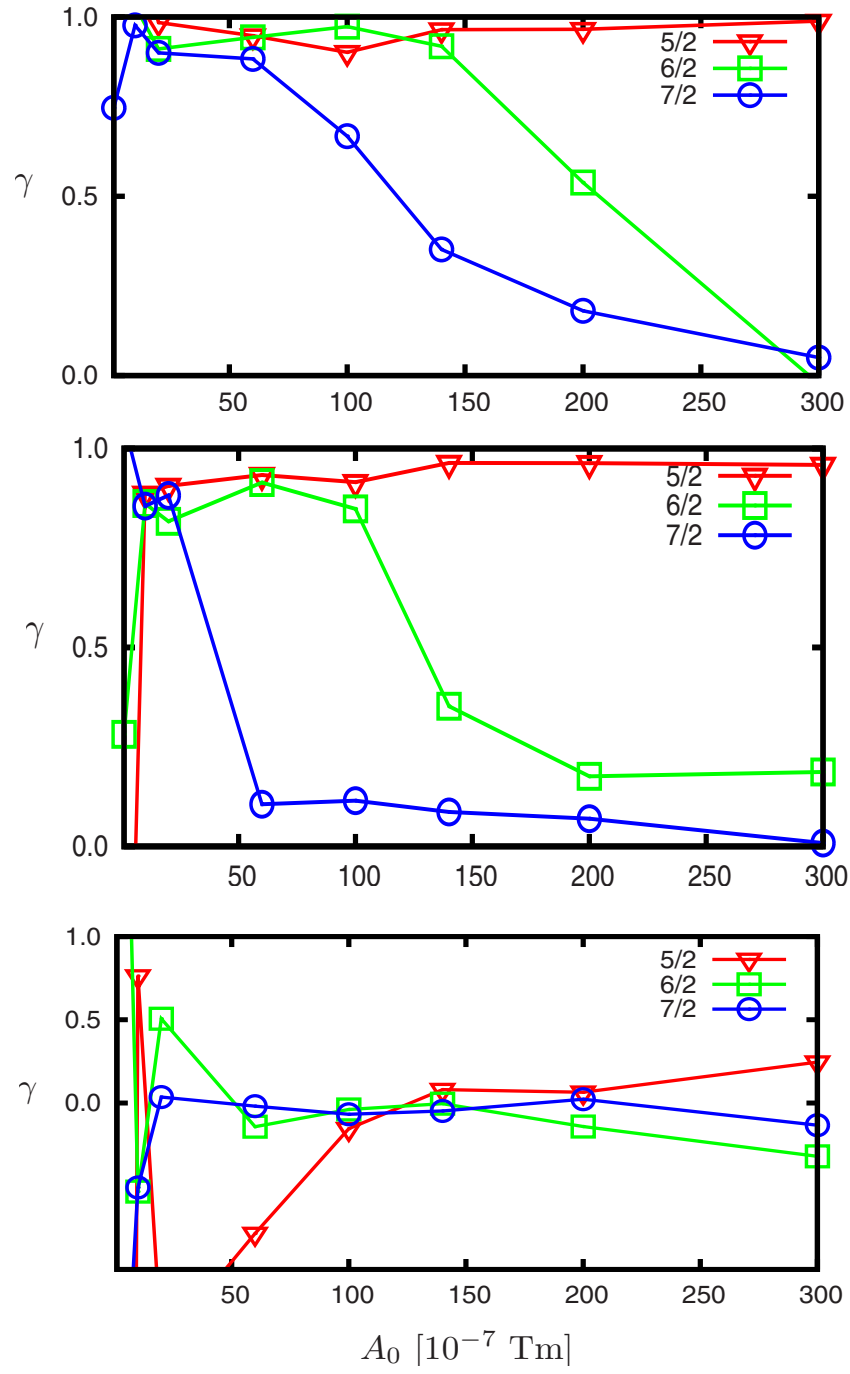

FIG. 7. (Color online) Screening efficiency at the respective resonant surfaces of the 5/2-, 6/2-, and 7/2-modes vs RMP field strength for $\hat{\beta}=5.0$ and $\hat{C}=5.0$ and for different model reductions.

by Fig. 7, bottom. Without pressure forces the plasma current is strongly reduced and consequently almost no screening effect is left (for some RMP amplitudes actually an enhancement of the RMP field is seen). Thus, only the inclusion of pressure forces provides a significant build-up of screening currents with the appropriate mode structure and the drop of shielding with increasing RMP.

\section{DISCUSSION}

In the numerical simulations the following observations are made:

- For TEXTOR relevant plasma parameters an RMP field can trigger a strong plasma response, such that induced currents modify the RMP vacuum field topology. A strong screening can hinder RMP fields from penetration and outof-phase currents can introduce additional topological structures. Such currents are directly related to important plasma dynamics such as GAMs and blobs.
- The shielding effect, preventing the RMP island chains to open, strongly depends on the ratio $\hat{C} / \hat{\beta}$ of collisionality and plasma beta.

- A threshold is observed in the dependence of the shielding effect on the RMP field strength. Up to a certain level of the RMP the screening is almost complete, but for higher RMP strength the screening breaks down abruptly. The absence of screening is of importance for the validity of models based on the vacuum approximation to analyze the topological features of plasma dynamics in RMP fields.

- In the simulations the response currents do not vary strongly with the RMP field strength, but the radial force balance $\partial\langle\bar{h}\rangle / \partial \chi$ is obviously strongly correlated with the screening efficiency. The drop in the shielding for increasing RMP level is accompanied by an increase in an induced radial electric field (zonal flow). The zonal flow is governed by the pressure gradient and the screening breaks down when the electric field compensates the pressure force, i.e., $\left\langle\partial\left(n_{0}+n\right) / \partial \chi\right\rangle \approx\langle\partial \phi / \partial \chi\rangle$.

- It is shown that these effects are apparently influenced by the presence of toroidal curvature, whereas the parallel ion motion does not have a significant impact. However, the basic mechanism is found in the inclusion of pressure forces in the electron momentum balance. If these are not present, almost no screening appears.

- The overlap of the three island chains introduced by the model RMP field does not seem to have a significant impact on the plasma response at the resonant surfaces. This allows to conclude that the screening effect is guided to a large extent by local mechanisms and is less affected by ergodization.

The importance of the interplay between poloidal plasma flows and the screening efficiency for RMP fields is basically well known. The radial component of the RMP exerts a torque on the plasma at the rational surfaces and an electric field is induced to establish a new stationary state. ${ }^{22}$ Here the basic facts are recalled by inspection of a simplified model in the framework of the drift-fluid-model used. Based on the numerical results it can be concluded that the following stationary (in the simulations: time averaged) balance equations describe the dominant constituents of the equilibrium plasma state at the resonant surfaces

$$
\hat{C} \bar{J}_{\|}=-\hat{\beta}\left\{\bar{A}+A^{*}, n_{0}-\bar{\phi}\right\}
$$

$$
\{\bar{\phi}, \bar{w}\}=-\hat{\beta}\left\{\bar{A}+A^{*}, \bar{J}_{\|}\right\} .
$$

The bar denotes a time-average and temporal correlation in the nonlinearities is neglected, i.e., the equilibrium is determined predominantly by the parallel force balance $e n_{0} E_{\|}$ $+\nabla_{\|} p_{e}=e n_{0} \eta_{\|} J_{\|}$and the quasineutrality condition $\boldsymbol{\nabla} \cdot \mathbf{J}$ $=\boldsymbol{\nabla} \cdot\left(\mathbf{J}_{\mathrm{pol}}+\mathbf{J}_{\|}\right)=0$, with the static polarization current $\mathbf{J}_{\mathrm{pol}}$ $=m_{i} n_{0} \mathbf{v}_{E} \cdot \nabla \mathbf{v}_{E} / B$. The modification of the axisymmetric radial pressure profile (the equilibrium profile), usually appearing in the numerical simulations, has been neglected for simplicity. The analysis is done for a single mode RMP, 


$$
A^{*}=A_{c}^{*} e^{n_{*} k_{\eta} \chi} \cos \left(n_{*} k_{\eta} \eta\right),
$$

which is resonant at $\chi=0$, where $q=q_{0}$. The approximate balance equations (33) and (34) are fairly justified a posteriori by the numerical results and predict a plasma response current which is controlled by the ratio $\hat{\beta} / \hat{C}$. This reflects the basic fact that a high collisionality $\hat{C}$ suppresses the rise of a response current and that a high $\hat{\beta}$ enforces the magnetic flutter response which can provide the screening. In order to proceed in the simple analysis, the fields $\bar{J}_{\|}, \bar{\phi}$, and $\bar{A}$ are assumed to have the functional form

$$
\bar{f}=f_{0}(\chi)+f_{c} \cos n k_{\eta} \eta+f_{s} \sin n k_{\eta} \eta,
$$

with constant coefficients $f_{c}$ and $f_{s}$. Furthermore it is assumed that the axisymmetric component of the vorticity $w_{0}$, i.e., the zonal flow contribution, is dominant. The effects due to poloidal fields in the plasma response are neglected $(\partial \bar{A} / \partial \chi=0)$, thus restricting the analysis on radial magnetic plasma response only. Taking everything into account one obtains from Ohm's law, Eq. (33), the following set of equations:

$$
\begin{aligned}
& \hat{C} J_{0}=\frac{n_{*}^{2} k_{\eta}^{2} \hat{\beta}}{2} A_{c}^{*} e^{n_{*} k_{\eta} \chi} \phi_{s}, \\
& \hat{C} J_{c}=n_{*} k_{\eta} \hat{\beta} A_{s}\left(\frac{\partial n_{0}}{\partial \chi}-\frac{\partial \phi_{0}}{\partial \chi}\right), \\
& \hat{C} J_{s}=-n_{*} k_{\eta} \hat{\beta}\left(A_{c}+A_{c}^{*} e^{n_{*} k_{\eta} \chi}\right)\left(\frac{\partial n_{0}}{\partial \chi}-\frac{\partial \phi_{0}}{\partial \chi}\right),
\end{aligned}
$$

and from the quasineutrality condition, Eq. (34),

$$
\phi_{s} \frac{\partial^{3} \phi_{0}}{\partial \chi^{3}}=-\hat{\beta} A_{s} \frac{\partial J_{0}}{\partial \chi} .
$$

Using Eq. (37) and twice integrating Eq. (40) gives

$$
\frac{\partial \phi_{0}}{\partial \chi}=-\frac{n_{*} k_{\eta} \hat{\beta}^{2}}{2 \hat{C}} A_{s} A_{c}^{*} e^{n_{*} k_{\eta} \chi}
$$

By inserting $J_{c}=n_{*}^{2} k_{\eta}^{2} A_{c}, J_{s}=n_{*}^{2} k_{\eta}^{2} A_{s}$, and $\chi=0$ into Eqs. (38), (39), and (41), and introducing the abbreviations

$$
a=\frac{\hat{\beta}}{n_{*} k_{\eta} \hat{C}}, \quad b=\frac{n_{*} k_{\eta} \hat{\beta}^{2}}{2 \hat{C}},
$$

the following set of nonlinear equations is obtained for $A_{s}$, $A_{c}$, and $\partial \phi_{0} / \partial \chi$ at the resonant surface:

$$
A_{c}^{* 2}=\frac{1+a^{2}\left(\frac{\partial n_{0}}{\partial \chi}-\frac{\partial \phi_{0}}{\partial \chi}\right)^{2}}{a b\left(\frac{\partial n_{0}}{\partial \chi}-\frac{\partial \phi_{0}}{\partial \chi}\right)} \frac{\partial \phi_{0}}{\partial \chi},
$$

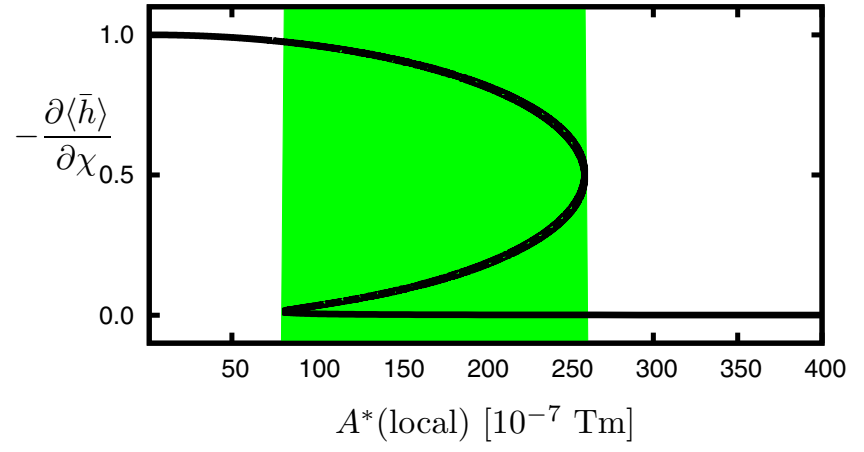

FIG. 8. (Color online) Functional relation of RMP level $A_{c}^{*}$ and radial electric field $\partial \phi_{0} / \partial \chi$, according to Eqs. (43) and (44). In the shaded region the solution for $\partial \phi_{0} / \partial \chi$ is not unique and a bifurcation takes place.

$$
A_{s}=-\frac{1}{b A_{c}^{*}} \frac{\partial \phi_{0}}{\partial \chi}, \quad A_{c}=a A_{s}\left(\frac{\partial n_{0}}{\partial \chi}-\frac{\partial \phi_{0}}{\partial \chi}\right),
$$

which must be fulfilled for a given set of values for $\hat{\beta}, \hat{C}$, and $A_{c}^{*}$. An immediate consequence of Eq. (43) is that the electric field must be in the range $\partial n_{0} / \partial \chi<\partial \phi_{0} / \partial \chi<0$. In addition, the relation (43) implies a bifurcationlike behavior for the radial electric field $\partial \phi_{0} / \partial \chi$ as a function of the RMP amplitude $A_{c}^{*}$. Figure 8 shows the possible solutions of $\partial \phi_{0} / \partial \chi$ for $0<A_{c}^{*}<4 \times 10^{-5} \mathrm{Tm}$ and $\hat{C}=\hat{\beta}=5.0$, i.e., the same parameters underlying the results of Figs. 5 and 7. It can be seen that for low and high RMP levels $A_{c}^{*}$ only one unique solution is found, i.e., $\partial \phi_{0} / \partial \chi \approx 0$ and $\partial \phi_{0} / \partial \chi \approx-1$, respectively. However in between these two limiting cases a transition region exists, where three solutions for the radial electric field are possible. This is a typical result for a bifurcation mechanism. The electric field can jump suddenly to another solution branch and a hysteresis is possible, dependent on the way the RMP field is changing. In view of this result the breakdown of the shielding effect observed in Figs. 5 and 7 can be understood as a consequence of a bifurcation process where the induced plasma currents and electric fields rearrange abruptly when parameter $A_{c}^{*}$ approaches a certain threshold. Of course the transition occurring in the results shown does not show a sudden jump, rather a smooth transition which is caused by additional plasma dynamics not included in the derivation of Eqs. (43) and (44). These model equations would allow to estimate quantitatively the RMP level in the transition range. Unfortunately this cannot be achieved in the framework of such an oversimplified model. Comparison with the numerical results shows that the threshold for the transition from complete shielding to almost complete RMP penetration does not reproduce the right dependence on plasma beta $\hat{\beta}$ and collisionality $\hat{C}$. This indicates that radial profile effects such as density profile flattening, induced poloidal fields, etc., have to be taken into account to obtain a reliable quantitative result. In addition, the modification of the screening mechanism due to curvature effects is shown to be significant, and this has been neglected completely in the simple analysis. Nevertheless, the simple estimate provides some insight into the physics, even though one must be aware that a thorough analysis of the RMP penetration requires more sophisticated analytical treatments 
or a numerical approach (at least) like the one presented here, taking into account the details of the 3D plasma dynamics and equilibrium magnetic field topology. For very high RMP fields the vacuum approximation might be a reasonable approach to describe the magnetic field topology, but the results presented here prove that strong shielding of RMP fields occurs for RMP levels up to $10^{-2} \mathrm{~T}$ and plasma densities and temperatures in the range of $3 \times 10^{18}-3$ $\times 10^{19} \mathrm{~m}^{-3}$ and $50-100 \mathrm{eV}$. These are quite usual conditions, e.g., in TEXTOR-DED, and for such operational regimes one has to be aware of a significant self-healing plasma response preventing island structures to open.

\section{SUMMARY}

Numerical simulations of four-field dissipative drift-fluid dynamics have been carried out for TEXTOR-like geometry and plasma parameters. This approach extends previous studies by inclusion of plasma currents, two-fluid pressure effects and toroidal geometry. The plasma response in the presence of static resonant magnetic perturbations has been studied for different plasma parameters and different levels of RMP field strength. The basic result is the occurrence of significant countercurrents, providing a shielding of the RMP field, such that a complete screening of the vacuum RMP field is possible. In addition out-of-phase currents occur which can have a strong impact on the resulting magnetic field structure, in such a way that the resulting confinement field structure is shifted by $\pi / 2$ in the toroidal direction compared to the externally induced RMP vacuum field. The vacuum approximation for the confinement field structure is not valid under such conditions. The screening depends strongly on the collisionality and the plasma beta, i.e., increasing $\hat{C}$ reduces the shielding, whereas increasing beta enhances the shielding effect. When increasing the RMP field amplitude for fixed plasma parameters one finds that the strong screening effect for low perturbation levels disappears and a new plasma state is established at higher perturbation levels where almost no screening of the RMP remains. The transition to the unscreened RMP field is relatively sharp and the threshold amplitude depends strongly on the plasma parameters. This transition is accompanied by the build-up of an electric field generating a static zonal flow in the vicinity of the resonant flux surfaces. The basic effects can be described quite well by simplified static versions of Ohm's law and the quasineutrality condition. On the basis of this analysis a bifurcation mechanism in the interplay of induced currents and poloidal plasma rotation could be identified. For increasing RMP levels the rising radial electric field tends to compensate the pressure force until the screening disappears. The simplified analysis could be justified by inspection of reduced models. This proves that parallel ion flow does not play an important role in the simulation results and pressure forces acting on the electrons are a prerequisite for the observed screening effect. Curvature does not change this effect qualitatively but have a significant impact on the threshold value for the bifurcation like breakdown of RMP shielding. With regard to the plasma parameters in the simulations one can conclude that for common tokamak scenarios and moderate RMP field levels (and below), the self-healing effect of the plasma can alter the vacuum structure of externally induced magnetic fields quite strongly.

\section{ACKNOWLEDGMENTS}

The authors would like to thank Professor P. Beyer for helpful discussions. One of the authors (D.C.) also gratefully acknowledges financial support by the European Commission for his stay at IEF-4 in Forschungszentrum Jülich GmbH, Germany.

\section{APPENDIX: SCALING AND PARAMETERS}

The scaling to obtain the dimensionless form equations (19)-(23) is chosen as

$$
\begin{aligned}
& \phi \rightarrow \frac{\rho_{s} T_{e}}{e L_{\perp}} \phi, \quad n \rightarrow \frac{\rho_{s} n_{0}}{L_{\perp}} n, \\
& A \rightarrow \frac{\rho_{s}^{2} q_{0} R_{0} \beta B_{0}}{L_{\perp}^{2}} A, \quad u \rightarrow \frac{\rho_{s} q_{0} R_{0} c_{s}}{L_{\perp}^{2}} u, \\
& J \rightarrow \frac{\rho_{s} q_{0} R_{0} e n_{0} c_{s}}{L_{\perp}^{2}} J, \quad t \rightarrow \frac{L_{\perp}}{c_{s}} t, \\
& \nabla_{\perp}^{2} \rightarrow \frac{\nabla_{\perp}^{2}}{\rho_{s}^{2}}, \quad \nabla_{\|} \rightarrow \frac{\nabla_{\|}}{q_{0} R_{0}}, \\
& \mathcal{K} \rightarrow \frac{\mathcal{K}}{B_{0} L_{\perp} \rho_{s}}, \quad B \rightarrow B_{0} B .
\end{aligned}
$$

The quantities $n_{0}$ and $q_{0}$ represent a density and pitch parameter at the reference radius $r_{0}$. The parameter $L_{\perp}$ denotes a typical background radial gradient length. The major radius is denoted by $R_{0}$ and $B_{0}$ is the reference magnetic field. The other quantities have their usual meaning.

${ }^{1}$ Ph. Ghendrih, A. Grosman, and H. Capes, Plasma Phys. Controlled Fusion 38, 1653 (1996)

${ }^{2}$ K. H. Finken, S. S. Abdullaev, A. Kaleck, and G. H. Wolf, Nucl. Fusion 39, 637 (1999).

${ }^{3}$ S. S. Abdullaev, K. H. Finken, M. W. Jakubowski, S. V. Kasilov, M. Kobayashi, D. Reiser, M. A. Runov, and R. Wolf, Nucl. Fusion 43, 299 (2003).

${ }^{4}$ A. B. Rechester and M. N. Rosenbluth, Phys. Rev. Lett. 40, 38 (1978).

${ }^{5}$ H. E. Mynick and J. A. Krommes, Phys. Fluids 23, 1229 (1980).

${ }^{6}$ R. J. Bickerton, Plasma Phys. Controlled Fusion 39, 339 (1997).

${ }^{7}$ A. Grosman, T. E. Evans, Ph. Ghendrih, E. Agostini, J. L. Bruneau, C. De Michelis, T. Fall, C. Gil, D. Guilhem, W. Hess, T. Hutter, J. Lassalle, G. Martin, M. Mattioli, P. Millot, P. Monier-Garbet, F. Mourgues, F. Nguyen, M. Paume, A. Samain, and J. C. Vallet, J. Nucl. Mater. 176-177, 493 (1990).

${ }^{8}$ T. E. Evans, R. A. Moyer, K. H. Burrell, M. E. Fenstermacher, I. Joseph, A. W. Leonard, T. H. Osborne, G. D. Porter, M. J. Schaffer, P. B. Snyder, P. R. Thomas, J. G. Watkins, and W. P. West, Nat. Phys. 2, 419 (2006).

${ }^{9}$ T. E. Evans, K. H. Burrell, M. E. Fenstermacher, R. A. Moyer, T. H. Osborne, M. J. Schaffer, W. P. West, L. W. Yan, J. A. Boedo, E. J. Doyle, G. L. Jackson, I. Joseph, C. J. Lasnier, A. W. Leonard, T. L. Rhodes, P. R. Thomas, J. G. Watkins, and L. Zeng, Phys. Plasmas 13, 056121 (2006).

${ }^{10}$ Y. Liang, H. R. Koslowski, P. R. Thomas, E. Nardon, B. Alper, P. Andrew, Y. Andrew, G. Arnoux, Y. Baranov, M. Bécoulet, M. Beurskens, T. Biewer, M. Bigi, K. Crombe, E. De La Luna, P. de Vries, W. Fundamenski, S. Gerasimov, C. Giroud, M. P. Gryaznevich, N. Hawkes, S. Hotchin, D. Howell, S. Jachmich, V. Kiptily, L. Moreira, V. Parail, S. D. Pinches, E. 
Rachlew, and O. Zimmermann, Phys. Rev. Lett. 98, 265004 (2007).

${ }^{11}$ Th. Eich, D. Reiser, and K. H. Finken, J. Nucl. Mater. 290-293, 849 (2001).

${ }^{12}$ M. Z. Tokar', Phys. Plasmas 6, 2808 (1999).

${ }^{13}$ O. Schmitz, M. W. Jakubowski, H. Frerichs, D. Harting, M. Lehnen, B. Unterberg, S. S. Abduallaev, S. Brezinsek, I. Classen, T. Evans, Y. Feng, K. H. Finken, M. Kantor, D. Reiter, U. Samm, B. Schweer, G. Sergienko, G. W. Spakman, M. Tokar, E. Uzgel, R. C. Wolf, and the TEXTOR Team, Nucl. Fusion 48, 024009 (2008).

${ }^{14}$ M. Kobayashi, Y. Feng, F. Sardei, D. Reiter, K. H. Finken, and D. Reiser, Nucl. Fusion 44, S64 (2004).

${ }^{15}$ D. Harting, D. Reiter, Y. Feng, O. Schmitz, D. Reiser, and H. Frerichs, Contrib. Plasma Phys. 48, 99 (2008).

${ }^{16}$ P. Beyer, X. Garbet, and P. Ghendrih, Phys. Plasmas 5, 4271 (1998).

${ }^{17}$ P. Beyer, X. Garbet, S. Benkadda, P. Ghendrih, and Y. Sarazin, Plasma Phys. Controlled Fusion 44, 2167 (2002)

${ }^{18}$ Q. Yu, S. Günter, Y. Kikuchi, and K. H. Finken, Nucl. Fusion 48, 024007 (2008).

${ }^{19}$ Y. Kikuchi, K. H. Finken, M. Jakubowski, M. Lehnen, D. Reiser, G. Sewell, and R. C. Wolf, Plasma Phys. Controlled Fusion 48, 169 (2006).

${ }^{20}$ R. Fitzpatrick and T. C. Hender, Phys. Fluids B 3, 644 (1991).

${ }^{21}$ R. Fitzpatrick, Phys. Plasmas 5, 3325 (1998).
${ }^{22}$ R. Fitzpatrick, Nucl. Fusion 33, 1049 (1993).

${ }^{23}$ R. Fitzpatrick, Phys. Plasmas 2, 825 (1995).

${ }^{24}$ A. Cole and R. Fitzpatrick, Phys. Plasmas 13, 032503 (2006).

${ }^{25}$ B. D. Scott, Plasma Phys. Controlled Fusion 39, 1635 (1997).

${ }^{26}$ V. Naulin, Phys. Plasmas 10, 4016 (2003).

${ }^{27}$ B. D. Scott, New J. Phys. 4, 52 (2002).

${ }^{28}$ B. Scott, Phys. Plasmas 8, 447 (2001).

${ }^{29}$ D. Reiser and B. Scott, Phys. Plasmas 12, 122308 (2005).

${ }^{30}$ D. Reiser, Phys. Plasmas 14, 082314 (2007).

${ }^{31}$ B. Unterberg, C. Busch, M. de Bock, J. W. Coenen, K. H. Finken, S. Jachmich, M. W. Jakubowski, Y. Kikuchi, A. Krämer-Flecken, M. Lehnen, U. Samm, O. Schmitz, S. Soldatov, M. Z. Tokar', M. von Hellermann, R. C. Wolf, Y. Xu, and the TEXTOR Team, J. Nucl. Mater. 363-365, 698 (2007).

${ }^{32}$ A. Krämer-Flecken, S. Soldatov, H. R. Koslowski, and O. Zimmermann, Phys. Rev. Lett. 97, 045006 (2006).

${ }^{33}$ A. Krämer-Flecken, S. Soldatov, D. Reiser, and M. W. Jakubowski, Proceedings of the 35th EPS Conference on Plasma Physics, Hersonissos, 9-13 June 2008, ECA Vol. 32D, p. 2.016.

${ }^{34}$ D. Reiser, Proceedings of the 35th EPS Conference on Plasma Physics, Hersonissos, 9-13 June 2008, ECA Vol. 32D, p. 2.031.

${ }^{35}$ S. I. Braginskii, Rev. Plasma Phys. 1, 205 (1965). 